\title{
Dominant Contributions to Lateral Distribution Functions in Ultra-High Energy Cosmic Ray Air Showers
}

\author{
Hans-Joachim Drescher and Glennys R. Farrar
}

2nd November 2018

Center for Cosmology and Particle Physics

Department of Physics, New York University

4 Washington Place, New York, NY 10003

\begin{abstract}
In hadron induced air showers of highest energies $\left(\mathrm{E}>10^{18} \mathrm{eV}\right)$, the lateral distribution functions of electrons and muons are a superposition of many separate electromagnetic sub-showers, initiated by meson decay at different altitudes and energies. The lateral distribution function is the primary tool for reconstructing the energy of the primary in a UHE cosmic ray shower, so understanding it in detail is a prerequisite for having confidence in the energy determination. We analyze in this paper the dominant contributions to the ground level lateral distribution functions, as a function of the altitude and energy at which the sub-showers are initiated. Far from the core, the dominant contribution to the density of electrons comes from sub-showers initiated at low altitudes and low energies $(\mathrm{E}<100 \mathrm{GeV})$. The dominant sub-showers are initiated at large radial distance from the core and at a large angle with respect to the main shower axis. This demonstrates the need for careful treatment of low energy hadron physics modeling even for ultrahigh energy primaries.
\end{abstract}

\section{Introduction}

There are basically two methods to measure air showers induced by cosmic rays with an energy greater than $10^{18} \mathrm{eV}$. One is to measure the fluorescence light caused by charged particles passing through the atmosphere; the other is to sample the lateral distribution of charged particles on the ground. Theoretical predictions for both the longitudinal and lateral distributions of secondaries are produced by Monte Carlo simulation of the showers. Comparing theory and observation permits properties of the primary cosmic ray, such as its energy and composition, to be inferred. 
In this paper we study lateral distribution functions of electrons and muons. In particular we want to determine which altitudes and which regimes of energy of the hadronic part of an air shower are of primary importance in producing various components of the ultimate ground level lateral distribution. This is a step toward the larger goal of elucidating the sources of systematic uncertainties in theoretical predictions for shower properties.

\section{Air showers}

Atmospheric showers can be categorized into three elements, the hadronic part, the electromagnetic part and the muonic part. Basically, the hadronic part feeds the other two components, photons being mainly produced by $\pi^{0} / \eta$ decay and muons by the decay of charged pions and kaons. Once a photon is produced it initiates an electromagnetic sub-shower which evolves essentially independently of the rest of the shower. (With some low probability, a photon interacts hadronically in a photo-nuclear reaction, re-initiating a hadronic sub-cascade; this is neglected here.) Muons, once created, interact very little. For them the most important process is energy loss via ionization, and occasionally pair production or bremsstrahlung in the nuclear field of air molecules.

Let us call the photons and muons which are directly produced by hadronic decays, initiators, because they initiate a sub-shower of their kind. The questions we address here are: where are these initiators produced, what are their energies, and what are the energies of the hadronic reaction in which they were themselves created. This is crucial to understanding lateral distribution functions and especially what governs the particle densities at large distances from the shower core.

For electromagnetic sub-showers there are two classes of initiators: high energy photons produced at high altitudes and low energy photons produced at low altitudes because high altitude initiators must have sufficient energy for their secondaries to reach ground level before being absorbed and sub-showers initiated at low energy must be created at low altitude, to avoid being absorbed before reaching the ground. However the the relative importance of these classes of initiators, as a function of the radial distance of the observed particle from the sub-shower, and the dominant sources of lateral spreading and arrival-time delay, has not been systematically studied until now.

For muons the situation is simpler than for electromagnetic showers. High energy charged pions rarely decay due to their huge Lorentz factor, so only low energy components of the hadronic shower contribute significantly to muon production. Moreover Lorentz beaming means that lower energy particles have typically larger angles with respect to the shower axis, and therefore contribute more to the muon density at larger distance from the core, than do higher energy particles. For a given initiator energy and angle, simple geometry implies that a muon can propagate to larger distances from the core if it is produced at higher altitudes. Muons, in contrast to electrons and photons propagate without much absorption to the ground level. 
The air showers analyzed in this paper are simulated with a traditional Monte Carlo method, whose code will be released soon. The basic features of this model are the high energy hadronic model QGSJET [1], the low energy hadronic model GHEISHA [2] and the electromagnetic shower model EGS4 [3]. The model has also some new simulation techniques which speed up the computation of air showers considerably, but these are irrelevant for the present analysis. Therefore, the pure physics content (hadronic and electromagnetic modeling) is identical to the most commonly used option in the CORSIKA package [4]. We have directly confirmed the agreement of the results presented here with CORSIKA simulations.

\section{Initiators}

In the following we analyze the contributions to the lateral distribution functions of $5 \times 10^{19} \mathrm{eV}$ proton-induced vertical showers at a ground level altitude of $900 \mathrm{~m}$, averaging over 50 showers. The kinetic energy cutoffs for registering hadrons, muons, electrons/positrons and photons have been chosen to $0.05 \mathrm{GeV}, 0.05$ $\mathrm{GeV}, 1 \mathrm{MeV}$ and $1 \mathrm{MeV}$, respectively.

\subsection{Ground electrons}

For electrons at ground level, the results are best summarized in Fig. 1, which shows the relative contribution at three distances from the core as a function of the electromagnetic initiator's energy and production depth. This is just the probability that an electromagnetic sub-shower is initiated at a given altitude and energy. The altitude is plotted in units of vertical atmospheric depth, defined as $X(h)=\int_{h}^{\infty} \rho\left(h^{\prime}\right) d h^{\prime}, h$ being the altitude and $\rho(h)$ the density of air, according to a standard USGS atmosphere. The results are obtained as follows: for each electron arriving at a certain distance from the shower core we trace back the history of this particle, generation by generation, and record the energy and depth of production of the initiator (the last particle of the sub-shower which was not a hadron: in most cases, a photon from $\pi^{0} / \eta$ decay).

One recognizes two distinct regions of initiator energy and depth of production which play the dominant role in producing electrons, depending on the distance from the core. Closer to the core, the initiators come predominantly from high altitudes and have high energies, while far from the core the initiators are seen to be primarily produced at low altitude with low energy.

We can analyze this interesting behavior in greater detail by looking at the probability distributions as a function of energy and depth separately. Figure 2 shows the distribution of energies of electron initiators whose electromagnetic sub-showers contribute to the electron density at 4 different lateral distances. The area under each curve is normalized to 1 to get probability distributions. Close to the shower core the most important contributions come from high energies. At larger distances the contribution of low energy initiators becomes more and more important. Fig. 3 gives the probability distribution as a function 
of the altitude of the initiators. At large distances from the core the dominant contribution comes from relatively low altitudes, i.e. large depth. This confirms that the power of lateral spreading for each high energy electromagnetic cascade is limited. In fact, the greatest contribution at large lateral distance comes from low energy sub-showers which are emitted at a large angle with respect to the main shower axis, as seen in Fig. 6 and at a large distance with respect to the shower axis (Fig. 5). It is useful to read Figs. 3 and 5 together, to see at which altitudes the initiators are produced, and then to read off the mean distance from the shower axis. One realizes that high altitude initiators are produced close to the axis, and low altitude ones close to the point where the electrons reach the ground. This means that the low energy hadronic part of the shower is producing a significant portion of the lateral expansion.

Fig. 3 shows a very irregular structure below $300 \mathrm{~g} / \mathrm{cm}^{2}$. This is a statistical fluctuation caused by some events having a very energetic photon, which is consequently a sub-shower initiator for many electrons and photons at ground level.

We note that the results on electron initiators presented here suggest why analytic treatments of the lateral distribution function of the air showers of hadronic primaries using a formula like the NKG [5, 6] are unsatisfactory, even though the formula may perfectly describe an electromagnetic sub-shower. The implementation of such analytic approaches generally assumes that each subshower initiator has a small angle with respect to the shower axis. As we have seen, this is not correct when describing electrons and positrons at large distances from the shower core. Only an implementation of NKG with full consideration of the emission angle of each sub-shower initiator, without small angle approximation, and taking the correct intersection of the inclined sub-shower with the ground level, can be expected to give reasonable results.

\subsection{Ground photons}

Photons show similar behavior to electrons and positrons, since they are created in the same electromagnetic sub-shower. However, Figs. 6 and 7 reveal that the effect discussed above for electrons is much less pronounced. Photon initiators are produced higher in the atmosphere than electron initiators, even though they are part of the same electromagnetic sub-showers. This is probably due to the fact that photons do not lose energy by ionization loss, therefore low energy photons have a higher chance to propagate a large distance than electrons do.

\subsection{Ground muons}

The corresponding results for muons can be seen in Figs. 8 and 9. Their energy spectrum (Fig. 8) at the point of creation gets peaked at a lower energy as one selects muons arriving at larger distance from the shower axis, because a

lower energy makes larger emission angles more probable. As for the depth of creation, one can see in Fig. 9 that at high altitudes muon initiators contribute more at large distances from the core, but never to the extent of electromagnetic 
initiators. This is not surprising since the hadronic shower has to evolve down in energy in order for pions or kaons to decay.

A comparison of Figs. 9 and 10 shows that muons observed at large radial distance are preferentially but not exclusively produced close the the shower axis, propagating to larger distances due to their large angle of production with respect to the shower axis.

The small peak in Fig. 9 at $r=2000 m$ and very low altitudes may be a fluctuation or the onset of a low altitude contribution as observed for electron densities; however the phenomenon is much less pronounced and we do not investigate it further here.

\section{Hadronic reaction energy}

It is also interesting to investigate the energy of the hadronic reactions which are responsible for creating the sub-shower initiators. Figure 11 shows the energy of the projectile in the lab frame, of the hadron-air collision which creates the electron- or muon-initiator. It reveals that high energy hadronic physics governs the production of the electromagnetic component of the ground level shower close to the core, but for larger distances from the core the last hadronic reaction is in the low energy $(\mathrm{E}<100 \mathrm{GeV})$ regime.

Figure 12 shows that at large distances from the core, low energy reactions become increasingly important for determining particle densities. The top figure shows the total lateral distribution function of electrons plotted in a full line, while the part contributed by initiators which are created in an hadronic reaction with an energy $E_{\text {lab }}>100 \mathrm{GeV}$ is shown with a dashed line. The corresponding curves for muons are drawn as dotted (muon lateral distribution function) and dashed-dotted lines (contribution by high energy reactions with $E_{\text {lab }}>100$ $\mathrm{GeV}$ only). The bottom figure shows the ratios of the corresponding curves, for electrons and for muons. At $600 \mathrm{~m} 20 \%$ of the electron density comes from a low energy hadronic reaction; at $1 \mathrm{~km}$ this fraction is $40 \%$. The situation is even more pronounced in the case of muons, due to the comparatively long lifetime of charged mesons. Here the last hadronic reaction is in most cases a low energy reaction, even at small distances from the core.

These results shed new light on the importance of low energy hadronic modeling in air showers, an aspect which is often neglected in the literature. The impact of low energy shower modeling can be directly seen in Fig. 11. In the simulation program used for this study, we employed the low energy hadronic model GHEISHA to describe interactions of projectiles whose lab energy is less than $90 \mathrm{GeV}$, while at higher energies QGSJET is used. This follows the CORSIKA procedure, only differing by choosing the energy of transition between low and high energy hadronic models at $90 \mathrm{GeV}$ rather than $80 \mathrm{GeV}$. Fig. 11 shows that the projectile energy distributions show a break at $\log _{10}(E / \mathrm{GeV}) \approx 2$, especially for muons (lower figure) at large distance from the core. The sharp break is an artifact of a discontinuity in the modeling of the hadronic interactions, between QGSJET and the low energy hadronic model GHEISHA. Evidently, at 
the transition energy $E_{\text {lab }}=90 \mathrm{GeV}$, GHEISHA produces many more muons at large distances than QGSJET; this arises because the two models have different differential cross sections and particle production levels. (For examples of differences between these models in this energy region, see [7].) With correct modeling, the high and low energy models must match at the transition energy, in principle in every detail. What we see here is that at large radius, these details affect the predicted shower properties. We have checked, however, that the qualitative conclusion that low energy, low altitude initiators dominate for large radius electrons, is not an artifact of the non-smooth transition between low and high energy hadronic models.

It has already been pointed out that for much lower energy primaries the low energy hadronic model is important for muons measured at ground level. In Ref. [8, 9] it is shown that for $10^{15} \mathrm{eV}$ hadronic showers, the energy of the last hadronic reaction before producing a muon $\left(E_{\mu}>1 \mathrm{GeV}\right)$ is in $50 \%$ of all cases a low energy reaction with $E_{\text {lab }}<100 \mathrm{GeV}$. Refs. [10, 11] study the low energy hadronic model dependence by analyzing CORSIKA [4i simulations of $10^{15} \mathrm{eV}$ hadron induced showers with two different models at energies below $80 \mathrm{GeV}$ : GHEISHA [2] and UrQMD [12, 13]. The two models show differences in the energy spectrum of produced muons, but the overall number of muons stays approximatively the same in both cases [11]. This fact becomes important when measuring muons with different energy thresholds as shown in Ref. [10]. While possibly not surprising, the analysis presented here shows that the low energy hadronic model is also important for determining the lateral distribution function at large radius for ultrahigh energy cosmic ray showers.

\section{Mean arrival time of ground particles}

We have seen that in the case of lateral distribution functions of electrons, there are two distinct regions in altitude and energy which contribute differently as a function of distance from the shower axis. This gives rise to the question where the time delay of particles with respect to the shower front comes from. In a simplified model one can consider three contributions: The time delay is caused by

- hadronic showering,

- electromagnetic showering, and

- geometry.

Fig. 13 shows illustrates the different parts. The hadronic time delay is defined as $\Delta t_{\text {had }}=t_{A B}-\overline{A B} / c$ with $\overline{A B}$ being the distance from $\mathrm{A}$ to $\mathrm{B}$ and $t_{A B}$ being the actual time spent in the hadronic cascade prior to production of the initiator, i.e. going from point A (first interaction) to point B (production of the initiator, e.g. a photon or a muon). $\Delta t_{\mathrm{em}}=t_{B C}-\overline{B C} / \mathrm{c}$ is the corresponding time delay of the electromagnetic (or muonic) sub-shower. The total time delay 
is defined as $\Delta t_{\text {tot }}=t_{A B}+t_{B C}-\overline{A C} / c$. And finally the geometric time delay is $\Delta t_{\text {geo }}=(\overline{A B}+\overline{B C}-\overline{A C}) / c=\Delta t_{\text {tot }}-\Delta t_{\text {had }}-\Delta t_{\text {em }}$. A detailed Monte Carlo model permits keeping track of each propagating particle and hence computing the values $\Delta t_{\mathrm{tot}}, \Delta t_{\mathrm{had}}, \Delta t_{\mathrm{em}}$.

Fig. 14 shows the results, which are best understood by referencing also the figures of the previous sections giving production height and radius of the initiators. Ground electrons close to the core spend most of their time in the electromagnetic sub-shower, since for them the main contribution comes from high altitude high energy initiators; the hadronic part of their history is at high energy for which multiple collisions do not increase the path length significantly, so their hadronic time delay is small. Further away from the core the hadronic part of the time delay increases, becoming as important as the electromagnetic one at $1500 \mathrm{~m}$. The increasing hadronic time delay is correlated with an increasing time delay caused by geometry, since both the radius of production of the initiators and their average emission angle increases with radius of the observed electron. Thus at very large distances, geometry and hadronic time delay are the dominant contributions, and the electromagnetic spreading is roughly constant as we have seen earlier.

As for ground photons, the main time delay is caused by the electromagnetic sub-shower. The difference from electrons becomes clear when one remembers that photon initiators are higher in the atmosphere than electron initiators (see Figs. 3 and (7).

The time delay of muons is mainly caused by geometry and, though less important, the hadronic part of the shower.

\section{Conclusions}

We have analyzed in detail how electromagnetic and muonic sub-showers contribute to the overall lateral distribution functions of an ultrahigh energy cosmic ray air shower. At all distances from the core, ground level muons come mostly from low energy muons emitted at intermediate-high altitudes in the atmosphere. However, at large distances from the core $(>1000 \mathrm{~m})$, electrons come predominantly from low energy $\pi^{0} / \eta$ 's produced at low altitudes and large distance from the core, and with a large angle with respect to the shower axis. Moreover the collisions producing the initiators are themselves low energy hadron-nucleus interactions which in standard air shower simulations are treated by a different model than the high energy interactions.

We conclude that in order to achieve detailed agreement between data and models for the lateral distribution functions and arrival-delay time distributions, necessary in order to optimally reconstruct properties of the primary, it is necessary to take greater care for modeling the low energy hadronic interactions in the showers. 


\section{Acknowledgments}

This research was supported by NASA grant NAG-9246, NSF-PHY-9996173, and NSF-PHY-0101738. The computations were made on NYU's Mafalda: a Linux cluster financed in part by the Major Research Instrumentation grant NSF-PHY-0116590.

We would like to thank A.A. Watson and R. Engel for useful comments on the first draft, as well as D.Heck for further informations.

\section{References}

[1] N.N. Kalmykov, S.S. Ostapchenko, and A.I. Pavlov. Nucl. Phys. B, 17, 1997.

[2] H.Fesefeldt. The simulation of hadronic showers. PITHA 85/02, Aachen 1985.

[3] W. R. Nelson, H. Hirayama, and D.W.O. the EGS4 Code System. SLAC265, Stanford Linear Accelerator Center, 1985.

[4] D. Heck, J. Knapp, J.N. Capdevielle, G. Schatz, and T. Thouw. Report FZKA 6019, 1998.

[5] K. Kamata and J. Nishimura. Progr. Theor. Phys., Suppl. 6, 1958.

[6] K. Greisen. Prog. Cosmic Ray Physics, 3, 1956.

[7] S.J. Sciutto. The Aires system for shower simulations. In Prceedings of the 27th International Comsmic Ray Conference.

[8] R. Engel. rapporteur talk: Particle and interaction physics. In proceedings of the 27th International Cosmic Ray Conference, Hamburg, Aug. 7-15, 2001.

[9] R. Engel, T.K. Gaisser, and T. Stanev. Extensive air showers and hadronic interaction models. In I. Sarcevic and C.-I. Tan, editors, proceedings of ISMD99 - International Symposium on Multiparticle Dynamics, Providence, Rhode Island, August 9-13, 1999. World Scientific.

[10] A. Haungs et al. The primary energy spectrum of cosmic rays obtained by muon density measurement at kascade. In proceedings of the 27th International Cosmic Ray Conference, Hamburg, Aug. 7-15, 2001.

[11] S. P. Swordy et al. The composition of cosmic rays at the knee. astroph/0202159, 2002.

[12] S. A. Bass et al. Microscopic models for ultrarelativistic heavy ion collisions. Prog. Part. Nucl. Phys., 41:225-370, 1998. 
[13] M. Bleicher et al. Relativistic hadron hadron collisions in the ultra- relativistic quantum molecular dynamics model. J. Phys., G25:1859-1896, 1999 . 

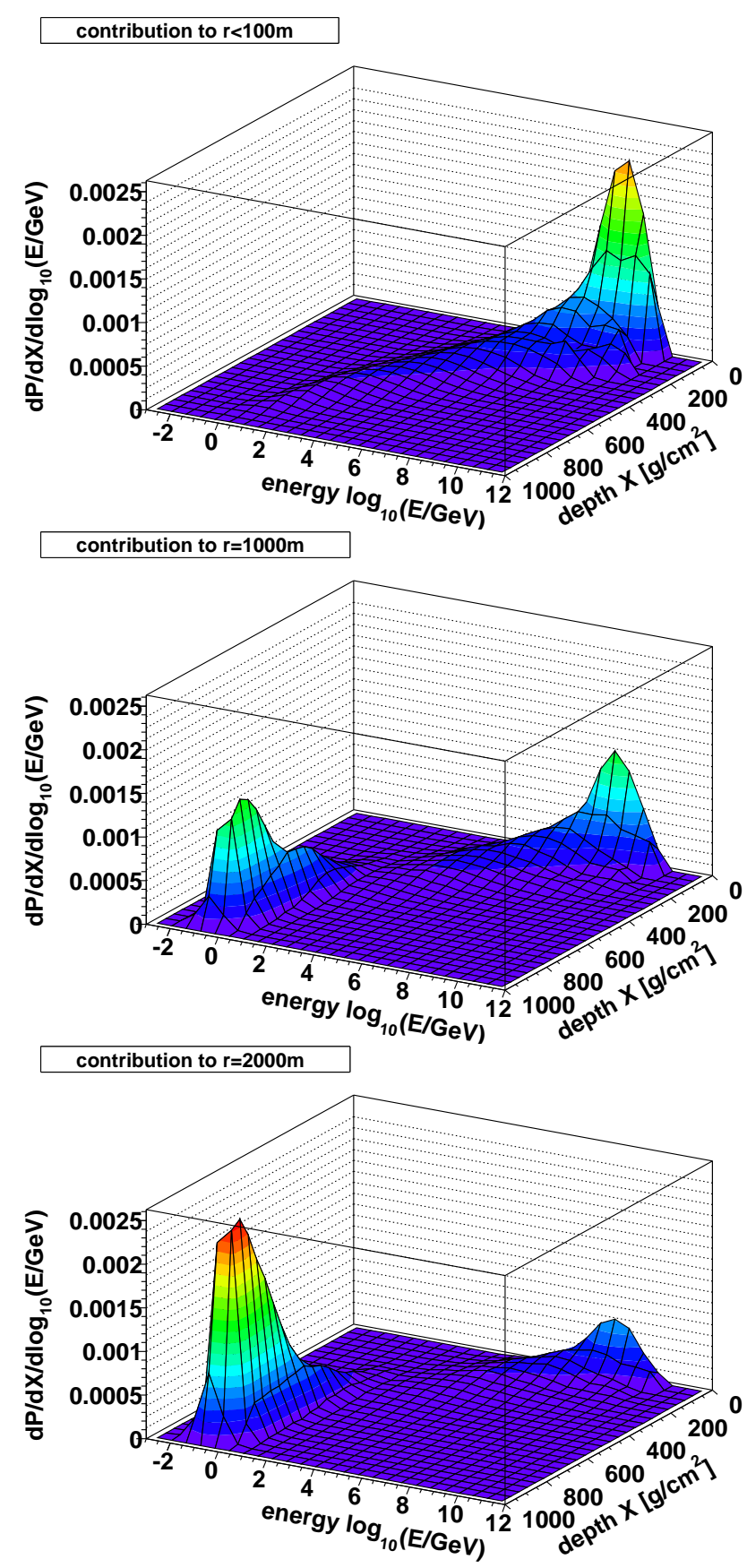

Figure 1: The relative contribution to electron densities at three distances from the core $\mathrm{r}<100, \mathrm{r}=1000$, and $\mathrm{r}=2000 \mathrm{~m}$, as a function of energy and production depth of the initiator of the electromagnetic sub-shower. Two distinct regions are visible, one at low energy and low altitude, and another at high altitude and high energy. 

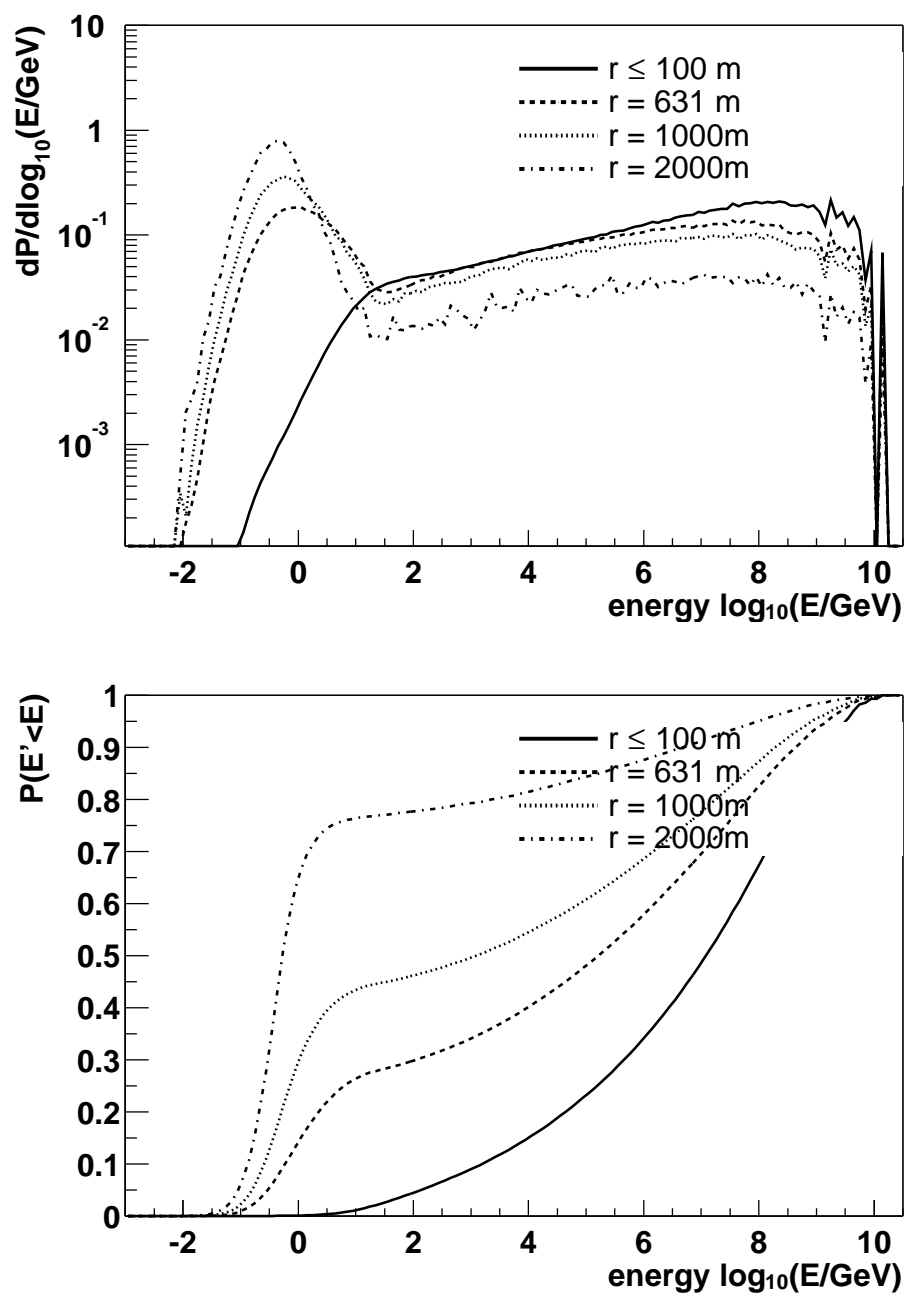

Figure 2: The top figure shows the probability distribution of initiators for ground level electrons as a function of the initiator's energy for different radial distances from the core. The bottom figure shows the integrated probability $P\left(E^{\prime}<E\right) \equiv \int_{0}^{E} \frac{d P}{d E^{\prime}} d E^{\prime}$ which corresponds to the fraction of initiators with energy less than $E$. At $\mathrm{r}=2000 \mathrm{~m} 65 \%$ of the electron density comes from low energy gammas with $E^{\prime}<1 \mathrm{GeV}$. 


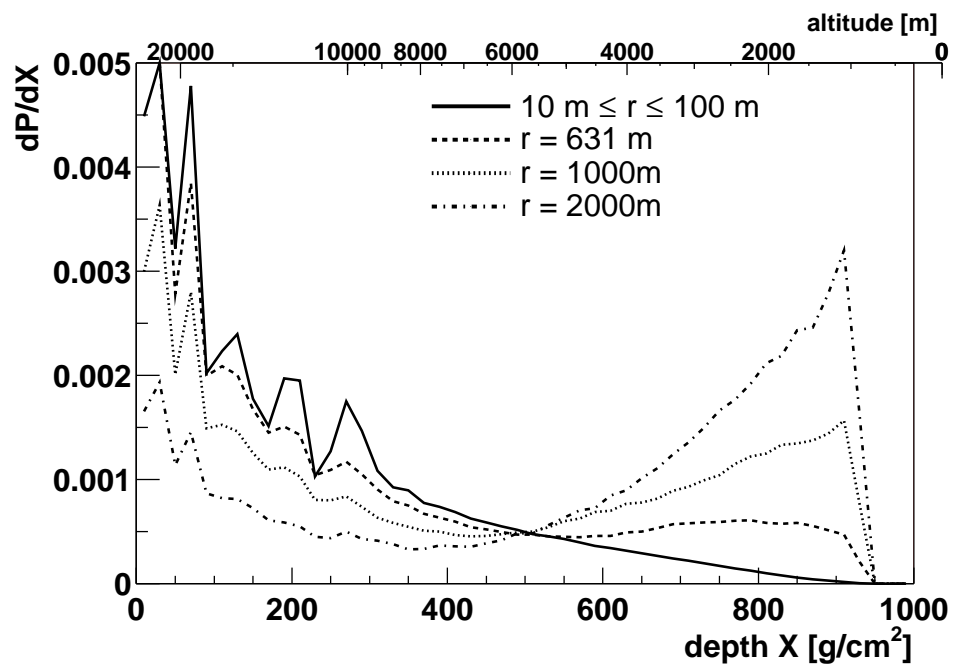

Figure 3: The depth of electron initiators for 4 different distances from the shower core. The greater is the distance from the core, the larger the contribution from low altitudes.

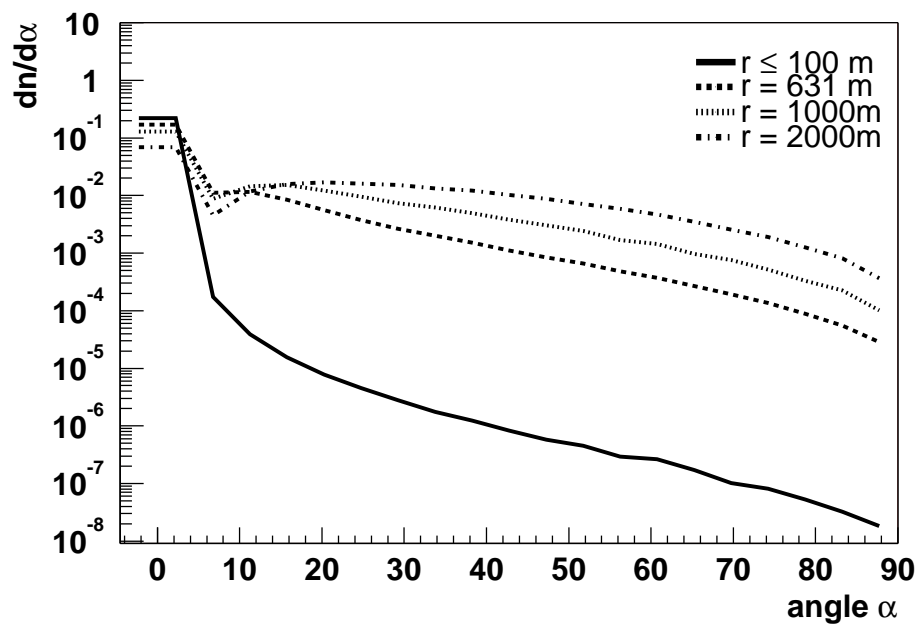

Figure 4: The emission angle of electron initiators as angle with respect to the main shower axis. The first bin has been duplicated in negative values for better visibility. Close to the core, the initiators are predominantly emitted collinear with the shower axis, while at larger distances greater emission angles are dominant. 


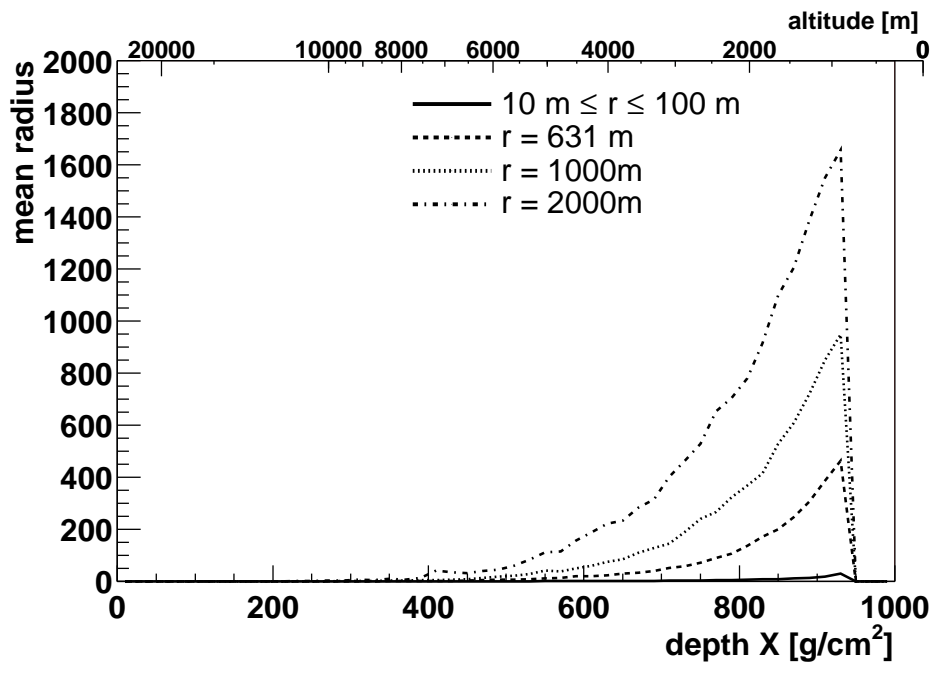

Figure 5: The mean distance of electron initiators to the shower axis.

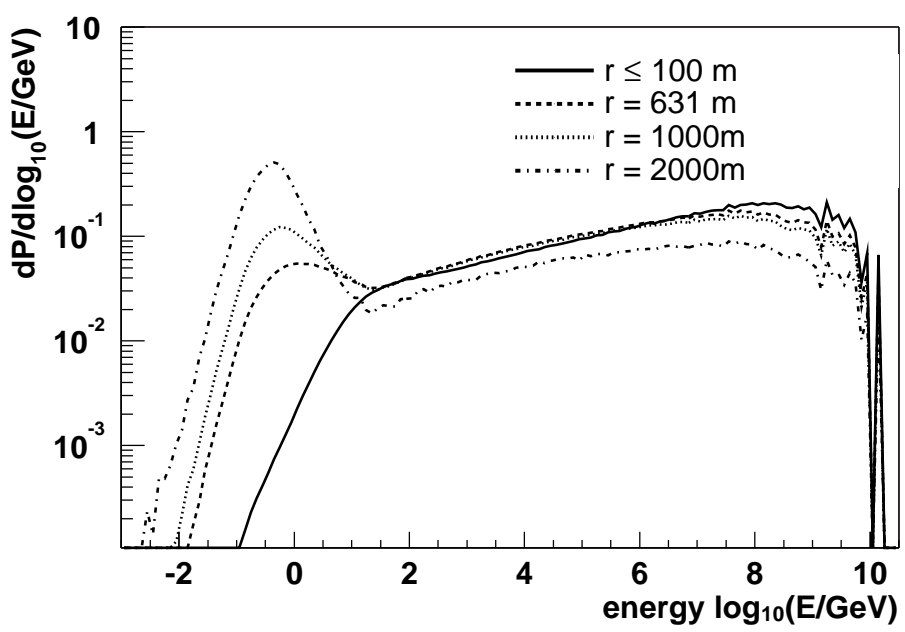

Figure 6: Energy distribution of photon initiators. 


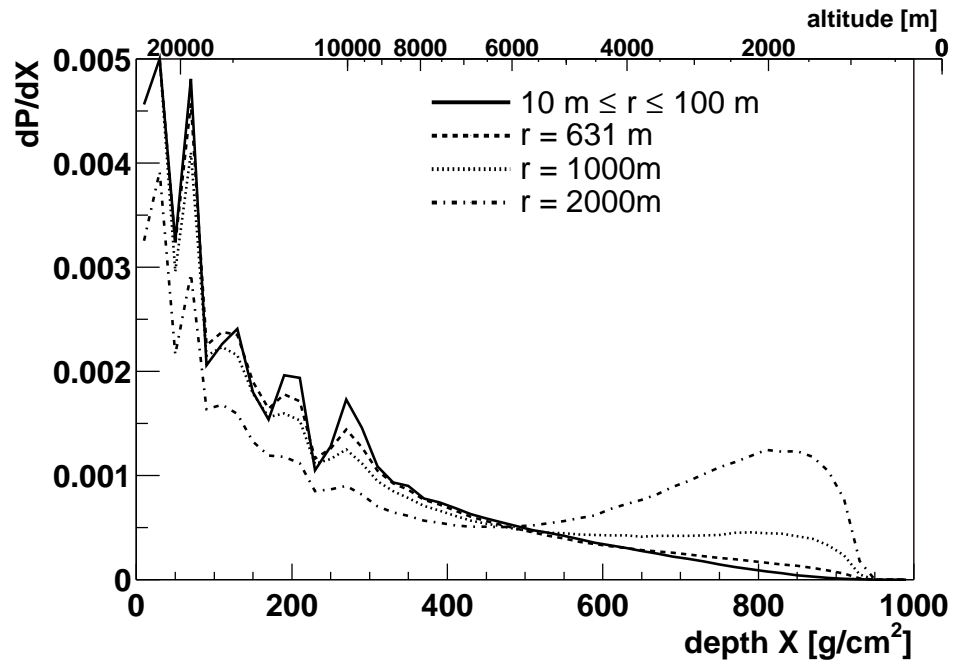

Figure 7: The distribution of vertical depth of photon initiators.

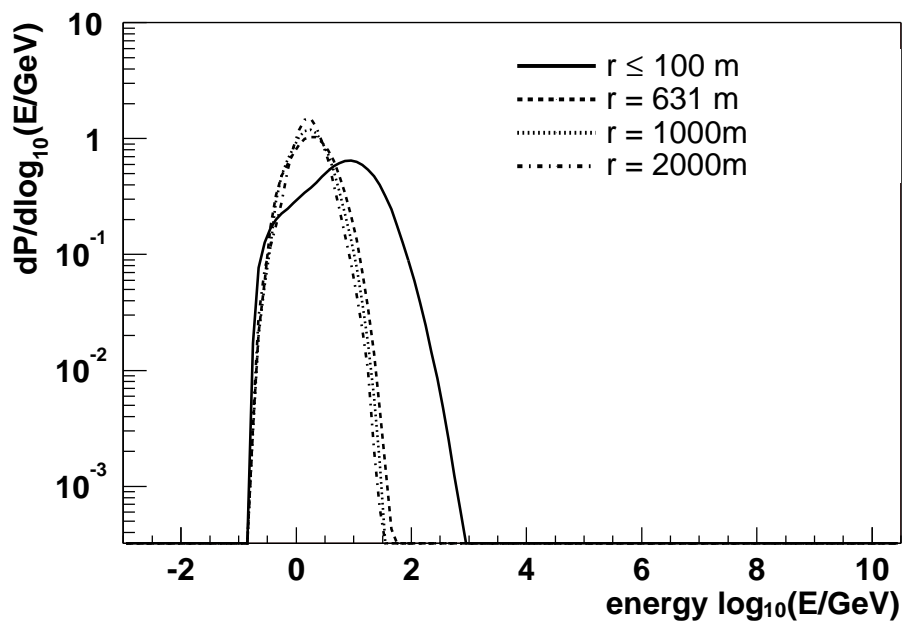

Figure 8: This plot shows the energy distribution of muon initiators, which is the energy of muons at the point of creation, leading to ground level muons at different lateral distances. 


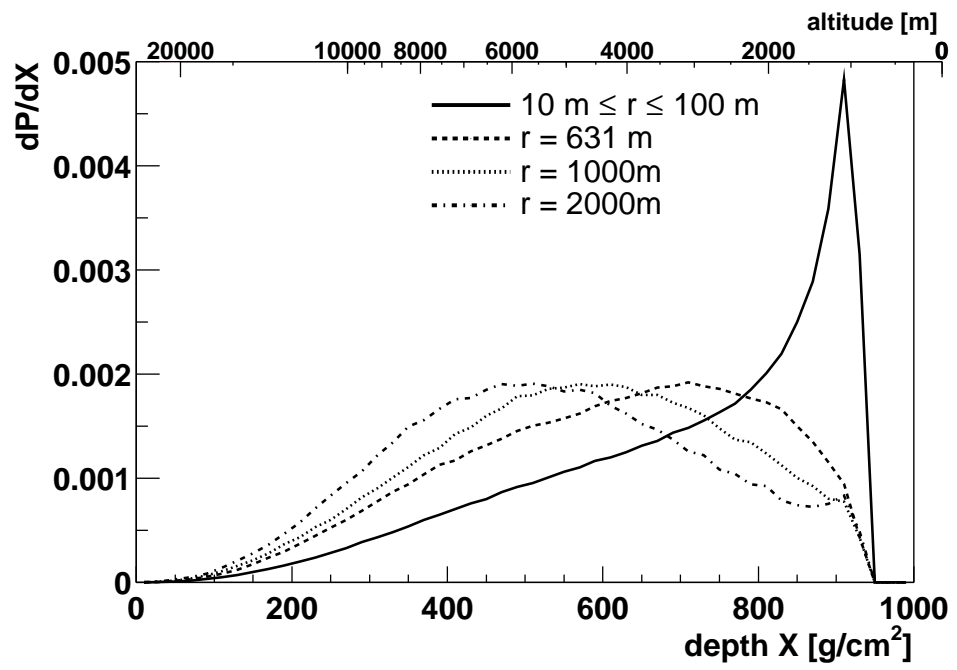

Figure 9: The depth of muons at the point of creation.

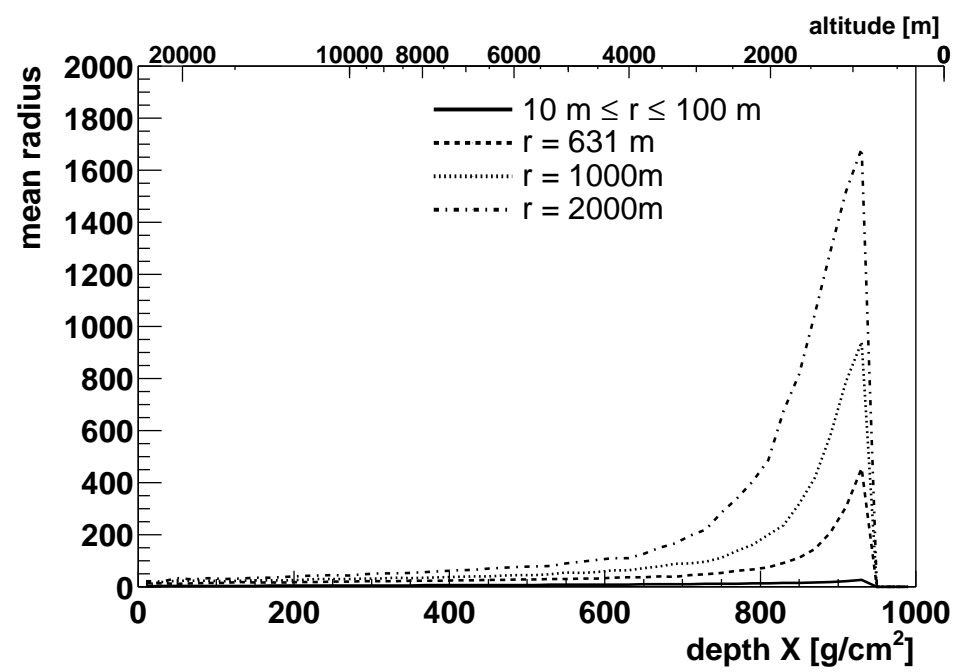

Figure 10: The mean distance of muon initiators to the shower axis. 

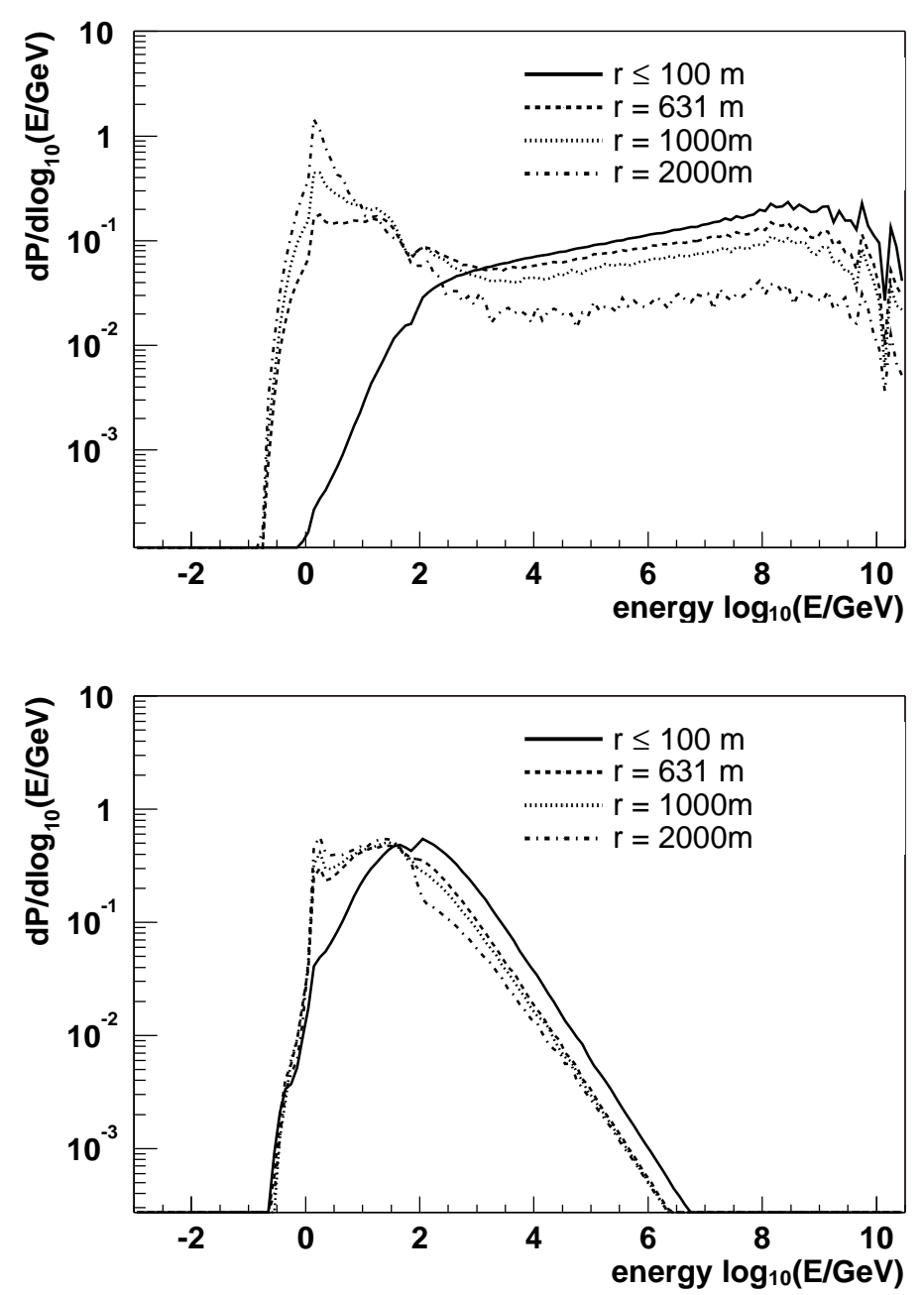

Figure 11: Energy of the last hadronic reaction before creating a electromagnetic (top) and a muonic (bottom) sub-shower. 

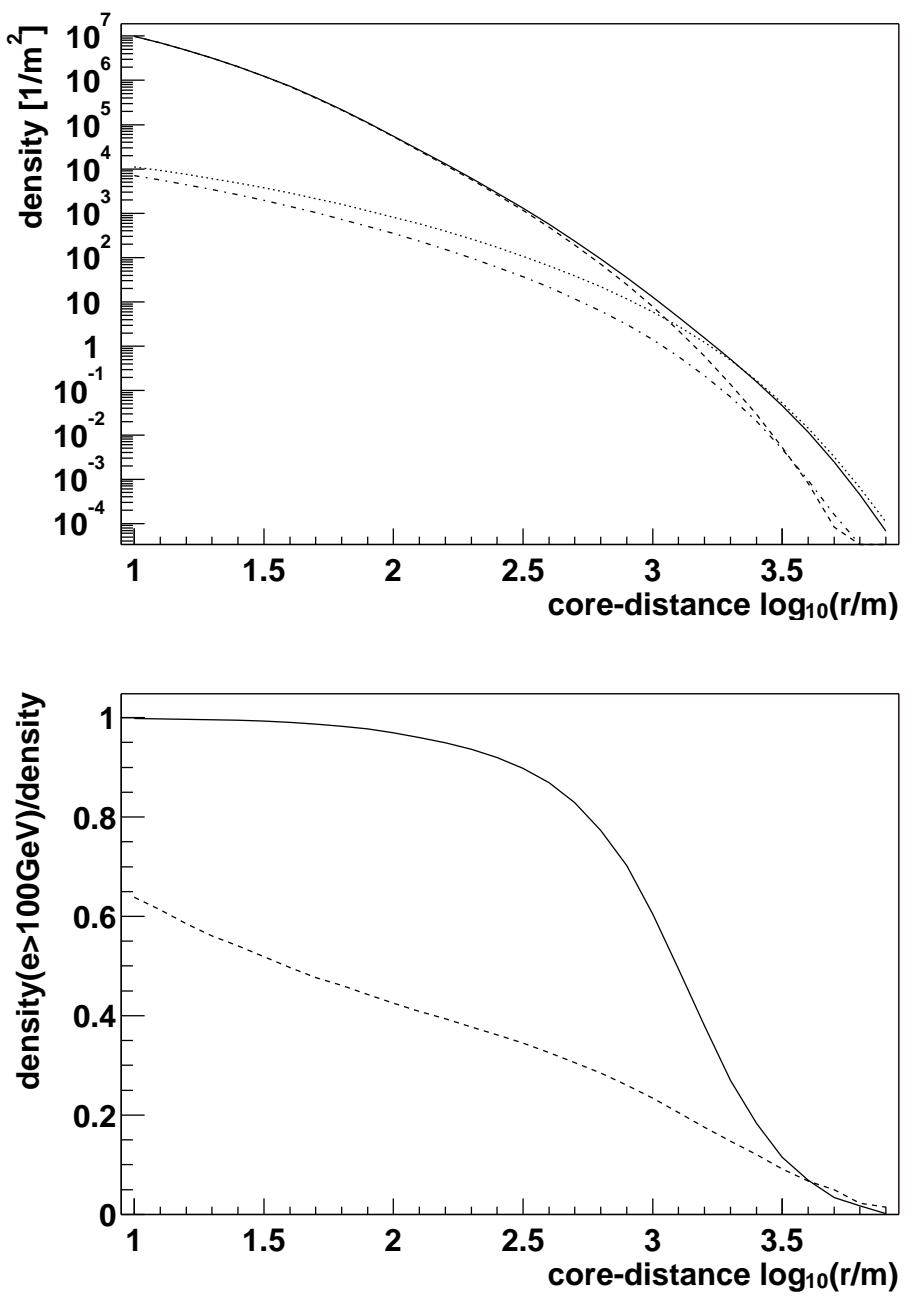

Figure 12: The importance of high energy reactions to the density of electrons and muons as a function of distance from the shower core: the full line in the top figure shows the total density of electrons as a function of distance from the shower core. The dashed line represents the density of electrons whose initiators are created in a high energy reaction $\left(E_{\text {lab }}>100 \mathrm{GeV}\right)$. The corresponding curves for muons are plotted as dotted and dashed-dotted lines. The bottom figure shows the ratios for electrons (full line) and muons (dashed line). 


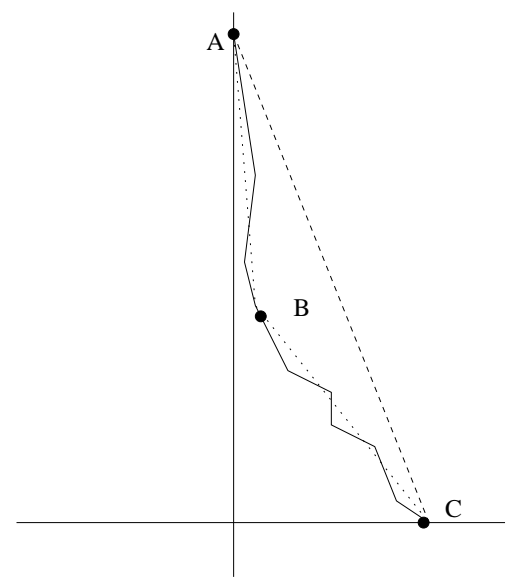

Figure 13: The contributions to the time delay of particles: hadronic time delay from A to B, electromagnetic/muonic from B to C. The difference of the sum (dotted line) to the total time delay (dashed line) represents the geometric delay as explained in the text. 

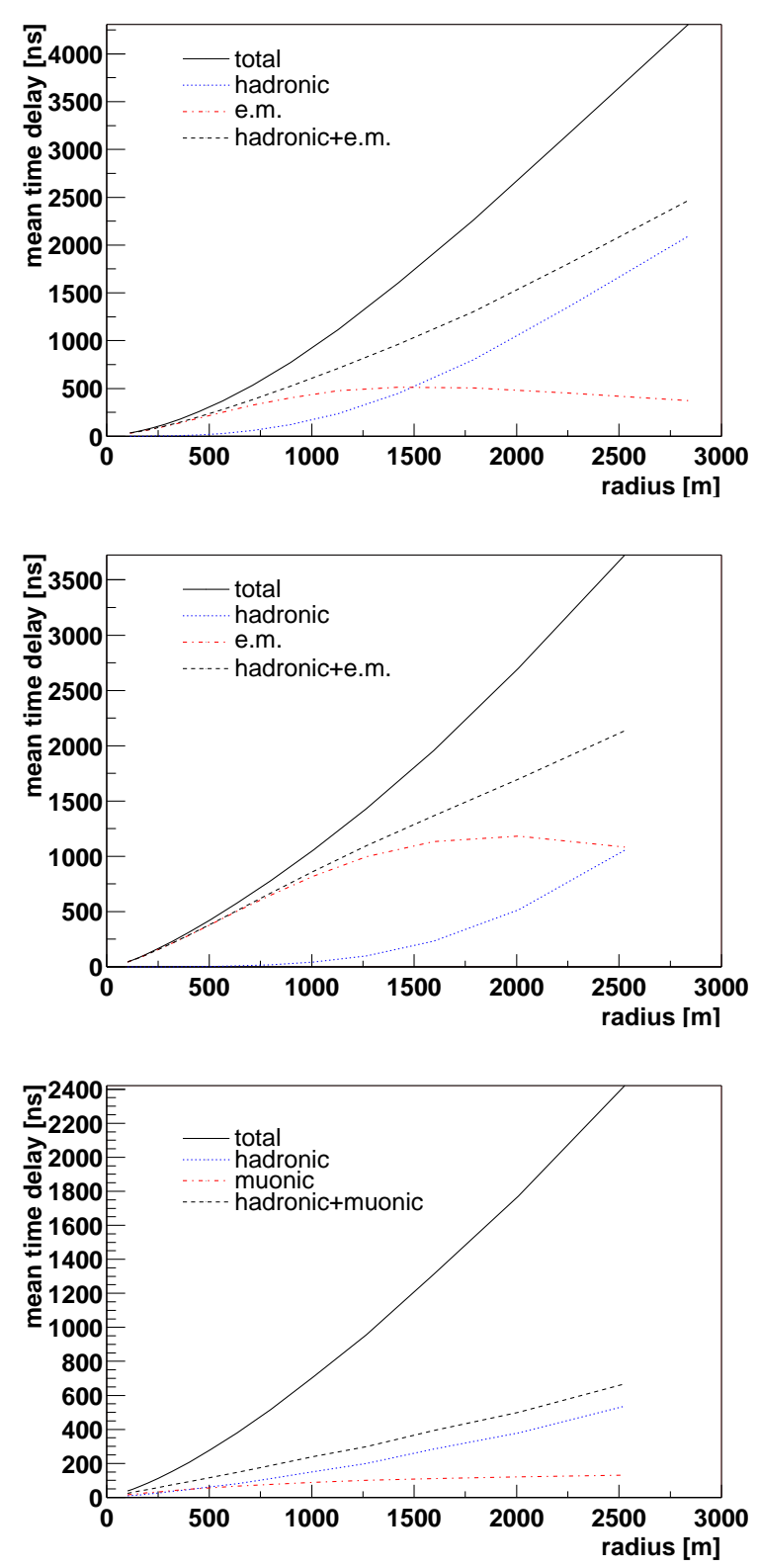

Figure 14: Contributions to the mean time delay of electrons (top), photons (middle) and muons (bottom) as a function of the distance from the shower axis. 